\title{
On Heteroscedastic, Skewed and Leptokurtic Log Returns and Spectral Density of Standardized Residuals
}

\author{
Ivivi Joseph Mwaniki \\ School of Mathematics, Division of statistics and actuarial Science, University of Nairobi, Kenya \\ Email: jimwaniki@uonbi.ac.ke
}

\begin{abstract}
A search for a distribution which adequately describes the dynamics of log returns has been a subject of study for many years. Empirical evidence has resulted in stylized facts of returns. Arguably, in this study, the three components of returns, mean equation part, the changing variance part and the resulting residuals are determined and their corresponding parameters estimated within the proposed framework. Spectral density analysis is used to trace the seasonality component inherent in the standardized residuals. Empirical data sets from eight different indexes and common stock are applied to the model, and results tabulated in support of the resulting framework.
\end{abstract}

Keywords: TGARCH, student t distribution, spectral density, seasonality.

\section{Introduction}

It is well known that the stock prices do not follow a pure random walk as documented in the literature. There exists a vast array of literature in search of a satisfactory descriptive statistical model for log returns. For example [1], proposed Poisson jumps with lognormal distributions, while [2], introduced a mixture of distribution hypothesis under a subordination process. Related modifications found in literature like in [3], ,4], [5] and [6] and many more confirms the need for such a model which might partially explain dynamics of log returns. Price changes are known to be neither independent nor identically distributed. There are linear and nonlinear dependencies between successive price changes. Distributional assumptions concerning risky asset log returns play a key role in option pricing and in risk management to say the least.

The presence of changing the second moment in returns were first captured in the ARCH model of [7] and later generalized to the GARCH model by [8]. Statistical properties of log returns have been compiled and documented as stylized facts. Most of those common stylized facts were like heteroscedasticity, gaussianity, heavy-tailed distribution can be found in [9] and [10] for example. One of those stylized facts indicates that empirical distributions of daily stock returns differ significantly from the traditional Gaussian model and tend to be skewed and leptokurtic with heavy-tailed distributions. Several distributions have been applied to model heavy-tailed aspect of returns, like hyperbolic distributions [11] , normal inverse Gaussian and other classes of generalized hyperbolic distribution [12], [13] and [14].

Recently, impacts of jumps in volatility and in returns, have been argued to influence dynamics of returns. Studies show that jumps in returns can generate a large movement such as the infamous crash of 1987, but the impact of a jump in volatility is transient, see [15],[16]. A jump in returns today has no impact on the future distribution of returns, on the other hand diffusive volatility is highly persistent, but its dynamics are driven by a diffusion process. While a jumps in returns are infrequent events, they are typically large and some empirical studies have shown that they explain 8 to $10 \%$ of the total variance in returns. Jump component, command relatively larger risk premia than the diffusive, one see [17],[18].

The main focus of this paper is to propose a general framework for modeling dynamics of the underlying process, incorporating some of the stylized features observed in log returns from different financial data sets, with an aim of tracing seasonal component. This paper is organized as follows. Section 2, presents a general framework of modeling log returns and a few examples (from literature) on the distribution of $\log$ returns and how they are nested within the proposed model. In Section 3 we discuss modelling of standardized residuals and the assumptions of covariance stationary to compute corresponding spectral densities. Parameter estimations, data analysis and summary of empirical results are presented in section 4. We draw conclusions and discussion in section 5 . 


\section{Modeling the Underlying}

\subsection{General Framework}

According to the the autoregressive jump intensity (hereafter ARJI) model by [19], the error term of $\log$ returns is specified to have two stochastic innovations of returns which comprise stochastic volatility component $\epsilon_{1, t}$ and a jump $\epsilon_{2, t}$. The first component, in this case $\epsilon_{1, t}$ is a zero mean innovation with a normal stochastic process, while $\epsilon_{2, t}$ is a jump innovation. These two innovations are contemporaneously independent. Similarly, we adopt [20] idea of modeling return (see also [21]) and extend it to get the following framework, which forms the basis of our empirical study.

Let $\left(\Omega, \mathcal{F},\left(\mathcal{F}_{t}\right)_{t \in[0, T]}, \mathbb{P}\right.$ be a stochastic basis describing the uncertainty of the economy. We refer to $\mathbb{P}$ as the physical probability measure and $\mathcal{F}_{t}$ represent the information flow driven by Brownian motion $B=\left(B_{t}\right)_{t \in[0, T]}$ and Lévy process $L=\left(\mathcal{L}_{t}\right)_{t \in[0, T]}$ of the stochastic process. Let $S_{t}$ be the price of a stock at time $t$ adapted to the natural filtration $\mathcal{F}_{t}$. Let $X_{t}$ be continuously compounded return on the stock.

Proposition 2.1. Define daily $\log$ return as $X_{t}=\log S_{t}-\log S_{t-1}$. Therefore

$$
X_{t}=\log \left(\frac{S_{t}+d_{t}}{S_{t-1}} \mid \mathcal{F}_{t-1}\right)
$$

where $d_{t}$ is one period dividends paid, then

$$
\begin{aligned}
\quad X_{t}= & m_{t}\left(. ; \theta_{\mathbf{m}}\right)+\sigma_{\mathbf{t}}\left(\vartheta_{\mathbf{t}}+\varpi \xi_{\mathbf{t}}\right), \\
\text { where } \quad & \xi_{t} \in \mathbf{G H} \forall t \in \mathbb{Z}^{+}, \quad \sigma_{t}^{2}=g\left(\sigma_{s}^{2}, \vartheta_{s} ; s \in(\infty, t-1] ; \theta_{\sigma}\right), \quad \varpi \geq 0, \\
& \vartheta_{t} \sim \text { i.i.d. }(0,1), \quad \xi_{t} \sim \text { i.i.d.D }\left(. ; \theta_{D}\right)
\end{aligned}
$$

$m_{t}\left(. ; \theta_{\mathbf{m}}\right)$ denote the mean function, $\sigma_{t}\left(. ; \theta_{\sigma}\right)$ denote the variance process and $\theta=\left(\theta_{\mathbf{m}}, \theta_{\sigma}, \theta_{\mathbf{D}}\right)$, and $\mathbf{G H}$ is a class of generalized hyperbolic distribution.

In equation $(1), m_{t}\left(. ; \theta_{\mathbf{m}}\right)$ denotes the conditional mean, which is governed by a set of parameters $\theta_{\mathbf{m}}$ provided that the process are measurable with respect to the information set $\mathcal{F}_{t-1}$, likewise the parameter set $\theta_{\sigma}$ governs the changing variance process, which depends on lagged values of innovations. $D\left(. ; \theta_{D}\right)$ is used to denote a zero mean distribution function which depends on the set of parameters in $\theta_{D}$.

\subsection{Examples Nested within the Framework}

Geometric Brownian motion As an example, let $S_{t}$ denote the price of the asset at time $t$ governed by geometric Brownian motion which solves the following SDE

$$
d S_{t}=\alpha S_{t} d t+\sigma S_{t} d W_{t}, \Rightarrow \frac{S_{t}}{S_{0}}:=e^{X_{t}}=\exp \left(\alpha t+\sigma W_{t}\right)
$$

where $\alpha$ and $\sigma$ are given constants. Let $h>0$ denote the length of time between observations of the price. Define $X_{t}(h)$ as a log return over the time interval $[t, t+h]$, then

$$
\begin{aligned}
d X_{t} & =\alpha d t+\sigma d W_{t}, \Rightarrow X_{t}=\alpha t+\sigma \sqrt{t} Z, Z \sim N(0,1), \text { thus } \\
X_{t}(h) & =\log _{e}\left(\frac{S_{t+h}}{S_{t}}\right)=\alpha[t+h-t]+\sigma\left(W_{(t+h)}-W_{t}\right) \\
& =\alpha h+\sigma W_{h}, \Rightarrow X_{t}(h) \sim N\left(\alpha h, \sigma^{2} h\right), \forall h>0 \\
\text { when } h=1 \quad X_{t} & =\alpha+\sigma Z, \text { where } m_{t}\left(., \theta_{\mathbf{m}}\right)=\alpha, \quad \sigma_{\mathbf{t}}\left(., \sigma_{\theta}\right)=\sigma, \vartheta_{\mathbf{t}}=\mathbf{Z}, \varpi=\mathbf{0} .
\end{aligned}
$$

It is well known that any normal distribution has zero skewness and excess kurtosis of zero, contrary to what we get from log returns. Attempts to model skewness and leptokurtic property of log returns, lend to augmenting geometric Brownian motion with Poisson driven jump process resulting to Bernoulli mixture jumps and jump diffusion model respectively. 
Bernoulli mixture jump process According to [4], the log returns are assumed to be driven by a Poisson mixture of jumps. Setting the mean size of jump equals to zero, it guarantees a symmetric distribution. Thus, the daily returns, whose density is a Bernoulli mixture of Gaussian densities is given by

$$
\begin{aligned}
f_{b}(x) & =\mathbb{P}[x=0] \phi\left(x, \alpha, \sigma^{2}\right)+\mathbb{P}[x=1] \phi\left(x, 0,\left(\sigma^{2}+\delta^{2}\right)\right) \\
& =(1-\lambda) \phi\left(\alpha, \sigma^{2}\right)+\lambda \phi\left(x, \sigma^{2}+\delta^{2}\right), \text { where } \\
\phi\left(\mu, \sigma^{2}\right) & =\frac{1}{\sqrt{\left(2 \pi \sigma^{2}\right)}} \exp \left(-\frac{(x-\mu)^{2}}{2 \sigma^{2}}\right) .
\end{aligned}
$$

This partially takes care of non normality aspect of heavy-tailed property of returns, but does not account for heteroscedasticity. This implies that

$$
m_{t}\left(., \theta_{\mathbf{m}}\right)=\mathbb{E}(\mathbf{x}):=\alpha^{*}, \quad \sigma_{\mathbf{t}}\left(., \sigma_{\theta}\right)=\operatorname{var}(\mathbf{x}):=\sigma^{*}, \quad \vartheta_{\mathbf{t}}=\mathbf{0}, \quad \varpi=\mathbf{1}, \quad \xi_{\mathbf{t}}=\mathbf{f}_{\mathbf{b}}(.)
$$

In this case the changing variance is assumed to be a constant. It can be seen quite easily that this process is nested in within the proposed framework.

Jump diffusion models In a stochastic differential form, the return process under the jump diffusion process, (see for example [3], [16], [22], [23], [24]) may be represented as,

$$
d S_{t}=\alpha S_{t} d t+\sigma S_{t} d W_{t}+J S_{t} d \pi(\lambda)
$$

where $\alpha$ is the drift; $\sigma$ the standard deviation of the diffusion component; $J$ is the jump size, and $\pi$ is a Poisson process with intensity parameter $\lambda$. Thus $X_{t}(h)$ is defined as

$$
X_{t}(h)= \begin{cases}x, & \text { if } K=0 \\ x+y_{1}+y_{2}+\ldots+y_{K}, & \text { if } K \geq 1\end{cases}
$$

$x \sim N\left(\mu h, \sigma^{2} h\right), y_{1}, y_{2}, \ldots, y_{k}$ is an i.i.d sequence with common distribution say $G$, while $K$ is distributed Poisson with parameter $\lambda h$. Therefore

$$
\begin{gathered}
\log _{e} S_{t+h}-\log _{e} S_{t}:=X_{t}(h)=\left(\left(\alpha-\frac{\sigma^{2}}{2}-k \lambda\right)+\sigma W_{t}+\sum_{i=1}^{N_{t}} \log _{e} Y_{i}\right), \forall h>0, \\
\text { if } h=1, X_{t}=\left(\alpha-\frac{\sigma^{2}}{2}-k \lambda\right)+\sigma\left(Z+\sum_{j=1}^{N_{t}} \log _{e} Y i\right), \text { where } \\
m_{t}\left(., \theta_{\mathbf{m}}\right)=\alpha-\frac{\sigma^{\mathbf{2}}}{\mathbf{2}}-\mathbf{k} \lambda, \quad \sigma_{t}\left(., \sigma_{\theta}\right)=\sigma, \quad \vartheta_{t}=Z, \quad \xi_{t}=\sum_{j=1}^{N_{t}} \log _{e} Y i .
\end{gathered}
$$

Empirical studies have documented much evidence as one of those stylized facts of conditionally changing variance, which led to many proposals of modeling varying jump intensities within the changing variance set up as studied by [6], and [25] for example.

\subsection{Modeling Changing Variance}

Several ARCH type models conditioned on either on Normal or student t distribution were used to model volatility. Specifications that could accommodate symmetric responses to negative and positive return innovations were considered. In order to relate to the flexible variance specifications in (1) to some existing model, we start by noting the functional format of $\operatorname{GARCH}(1,1)$ specification.

$$
\sigma_{t}^{2}=\omega+\alpha \sigma_{t-1}^{2}+\beta \epsilon_{t-1}^{2}
$$


Such models are generally said not to allow for leverage effect, which refers to the tendency for changes in stock prices to be negatively correlated with volatility. One such extension to the GARCH model considered in (4) is the nonlinear asymmetric GARCH model, or NGARCH model, of [26] given by

$$
\sigma_{t}^{2}=\omega+\alpha \sigma_{t-1}^{2}+\beta \sigma_{t-1}^{2}\left(\epsilon_{t-1}+\gamma\right)^{2}
$$

In the NGARCH model the leverage effect is modeled through the parameter $\gamma$, and if $\gamma<0$ then there is a leverage effect, otherwise if $\gamma=0$ the model turns out to be GARCH model. We observe that GARCH response is characterized by a symmetric response of volatility to positive and negative errors $\epsilon_{t-1}$. To model impacts of lagged positive and negative innovations one may favour a so called threshold GARCH which was proposed by [27] for the variance and by [28] for the standard deviation. The TGARCH $(1,1)$ model for $\sigma_{t}^{2}$ takes the following form

$$
\sigma_{t}^{2}=\omega+\beta \epsilon_{t-1}^{2}+\eta \epsilon_{t-1} \mathbb{I}_{\epsilon_{t-1}<0}+\alpha \sigma_{t-1}^{2}
$$

where $\mathbb{I}($.$) denotes an indicator function. The leverage effect describes that the current volatility is more$ affected by negative innovations relative to the positive ones. The following models were used to model the changing variance component with the intention of extracting a leptokurtic standardised residuals. GARCH(1,1), TGARCH(1,1) and $\operatorname{NGARCH}(1,1)$ specifications conditioned on standardized normal and student $t$ distributions respectively.

\section{Normal distribution and student $\mathrm{t}$ distribution}

If the conditional distribution $f\left(x_{t} \mid \mathcal{F}_{t-1}\right)$ is normal with mean $\mu_{t}$ and variance $\sigma_{t}^{2}$ the likelihood function is given by

$$
f\left(x_{1}, \ldots, x_{T} ; \theta\right)=f\left(x_{1} ; \theta\right) \prod_{t=2}^{T} \frac{1}{\sqrt{2 \pi \sigma_{t}}} \exp \left(-\frac{\left(x_{t}-\mu_{t}\right)^{2}}{2 \sigma}\right)
$$

where $f\left(x_{1} ; \theta\right)$ is the marginal density function of the first observation $x_{1}$. The value that maximizes the likelihood function given by

$$
€=\log _{e} f\left(x_{1}, \theta\right)-\frac{1}{2} \sum_{t=1}^{T}\left(\log _{e} 2 \pi+\log _{e} \sigma_{t}^{2}+\frac{\left(x_{t}-\mu_{t}\right)^{2}}{\sigma_{t}^{2}}\right)
$$

A similar case follows for the standardized student t-distribution. Let $x_{v}$ be a student t distribution with $v$ degrees of freedom. Then $\operatorname{var}(X)=v /(v-2)$ for $v>2$. Define $\epsilon_{t}=X / \sqrt{v / v-2}$. The probability density of $\epsilon_{t}$ is

$$
f\left(\epsilon_{t} \mid v\right)=\frac{\Gamma(v+1) / 2}{\Gamma(v / 2) \Gamma(v-2) \pi}\left(1+\frac{\epsilon_{t}^{2}}{v-2}\right)^{-(1+v) / 2}, v>2
$$

If we let, $a_{t}=\sigma_{t} \epsilon_{t}$, we obtain the conditional likelihood function of $a_{t}$ as

$$
\mathrm{E}=\log _{e} f\left(. \mid \alpha, A_{m}\right)=-\sum_{t=m+1}^{T}\left[\frac{v+1}{2} \log _{e}\left(1+\frac{a_{t}^{2}}{(v-2) \sigma_{t}^{2}}\right)+\frac{\log _{e} \sigma_{t}^{2}}{2}\right]
$$

\section{Leptokurtic Residuals and Their Spectral Density}

The optimal combination of ARMA-GARCH type models of each data set under investigation was used to model changing variance component (say, $\sigma_{t}$ of $(1)$ ) and the resulting estimates were as reported in Table 3. As a result, properties of the filtered standardized leptokurtic covariance stationary and skewed residuals, $\xi_{\mathbf{t}}$ of in (1) were investigated further, across different data sets, all of the residuals fit Normal inverse gaussian distribution. Moreover their spectral densities were determined and different markets data gave different spectral densities with different periodicity as reported Table 2. Spectral densities used are as defined in the following subsections. 


\subsection{Spectral Densities of Standardized Residuals}

For a properly specified ARCH model, the standardized residuals $\hat{a_{t}}=a_{t} / \sigma_{t}$ form a sequence of i.i.d random variable. Let $\left\{\gamma_{h}, h=0, \pm 1, \pm 2, \ldots\right\}$ be the autocovariance function of discrete stationary time series, then the power spectrum $P(v)$ of the series is defined as

$$
P(v)=\sum_{h=-\infty}^{\infty} \gamma(h) e^{-i v h}, \quad v \in[-\pi, \pi], \text { where } \sum_{h=-\infty}^{\infty}|\gamma(h)|<\infty
$$

The normalized spectral density function, say $f(v)$ is defined as the Fourier transform of the ACF (auto correlation function) $\rho_{h}=\gamma(h) / \gamma(0), h=0, \pm 1, \pm 2, \ldots$ is given by

$$
f(v)=\frac{1}{\pi} \frac{P(v)}{\gamma(0)}=\frac{1}{\pi} \sum_{h=-\infty}^{\infty} \frac{\gamma(h)}{\gamma(0)} e^{-i v h}, \quad \rho_{h}=\rho_{-k}, \quad v \in[-\pi, \pi]
$$

The spectrum of covariance stationary time series is a positive real function of a frequency variable associated with a stationary stochastic process. Intuitively the spectrum decomposes the content of a stochastic process into different frequencies in that process and helps us identify periodicity. We consider four different models and their corresponding normalized spectral densities, (i) pure white noise, (ii) the $\operatorname{AR}(1)$, (iii) $\operatorname{ARMA}(1,1)$ and (iv) seasonal autoregressive model hereafter (SAR(1)).

(i) Pure random process

Let, $Y_{t}=W_{t}$ where $\left\{W_{t}\right\}$ is pure white noise, it implies

$$
\begin{gathered}
\gamma(h)=\left\{\begin{array}{l}
\sigma_{w}^{2}, h=0 \\
0, \quad h>0 .
\end{array}\right. \\
\Rightarrow f_{y}(v)=\frac{1}{2 \pi} \sum_{h=-\infty}^{\infty} \gamma(h) e^{-2 \pi i v h}=\frac{\gamma(0)}{2 \pi}=\frac{\sigma_{w}^{2}}{2 \pi}, \quad v \in[-1 / 2,1 / 2] .
\end{gathered}
$$

We observe that a process is regarded as a practical approximation of a continuous white noise, if the spectrum is substantially constant over the frequency band of interest.

(ii) $\operatorname{AR}(1)$ process

Let $Y_{t}=\Phi Y_{t-1}+W_{t}$, where $W_{t}$ is a white noise and $|\Phi|<1$, then its auto covariance function is given by $\gamma_{k}=\operatorname{cov}\left(Y_{t}, Y_{t-k}\right)=\Phi \gamma_{k-1}=\Phi^{k} \gamma_{0}$ where $\gamma_{0}=\operatorname{var}\left(Y_{t}\right)$. This implies that $\gamma_{0}=\sigma_{w}^{2} /\left(1-\Phi^{2}\right)$. The spectral density for $\mathrm{AR}(1)$ is given by

$$
\begin{aligned}
f_{y}(v) & =\frac{1}{\pi} \sum_{h=-\infty}^{\infty} \gamma(h) e^{-2 \pi i v h}=\frac{1}{\pi} \sum_{h=-\infty}^{\infty} \frac{\sigma_{w}^{2} \Phi^{h}}{1-\Phi^{2}} e^{-2 \pi i v h}, v \in[-1 / 2,1 / 2] \\
& =\frac{\gamma_{0}}{2 \pi}\left(1+\frac{2}{\pi} \sum_{k=1}^{\infty} \Phi^{k}\left[e^{i 2 \pi v k}+e^{-i 2 \pi v k}\right]\right)=\frac{\gamma_{0}}{\pi}\left(1+\frac{\Phi e^{i 2 \pi v}}{1-\Phi e^{i 2 \pi v}}+\frac{\Phi e^{-i 2 \pi v}}{1-\Phi e^{-i 2 \pi v}}\right) \\
& =\frac{\gamma_{0}}{\pi} \frac{1-\Phi^{2}}{1-2 \Phi \cos 2 \pi v+\Phi^{2}}=\frac{1}{2 \pi}\left(\frac{\sigma_{w}^{2}}{1-2 \Phi \cos (2 \pi v)+\Phi^{2}}\right) .
\end{aligned}
$$

(iii) $\operatorname{ARMA}(1,1)$ Process

Similarly, if $Y_{t}=\Phi Y_{t-1}+W_{t}+\theta W_{t-1}, \quad|\Phi|<1, \quad|\theta|<1$, it follows that

$$
f_{y}(v)=\frac{\sigma_{w}^{2}}{2 \pi}\left(\frac{1+2 \theta \cos (2 \pi v)+\theta^{2}}{1-2 \pi \cos (2 \phi v)+\Phi^{2}}\right), \quad v \in[-1 / 2,1 / 2] .
$$

(iv) SAR(1) Process

A stationary first order seasonal autoregressive model $S A R(1)_{q}$ of $Y_{t}$, is given by

$$
Y_{t}=\Phi Y_{t-q}+W_{t}, \quad 0<\Phi<1 .
$$


Therefore $\left(1-\Phi \mathbb{L}^{q}\right) Y_{t}=W_{t}, \Rightarrow \psi(\mathbb{L})=\left(1-\Phi \mathbb{L}^{q}\right)^{-1}$ and its normalized spectral density is given by

$$
f(v)=\frac{\sigma_{w}^{2}}{2 \pi}\left(\frac{1}{1-2 \Phi \cos (2 \pi q)+\Phi^{2}}\right) .
$$

The resulting spectral density is periodic with period $1 / q$, and gets peaked as $e^{-2 \pi i v}$ passes near the roots of the complex function $1-\Phi_{1} z^{q}=0$ where $r=|\Phi|^{-1 / q}$ and $z=r e^{i \theta}, r=|\Phi|$. For example if $q=12$, then the twelve poles are at $|\Phi|^{-1 / 12} e^{q \pi i k / 6}$ where $k=0, \pm 1, \pm 2, \ldots, \pm 6$. Thus if $0<\Phi<1$ and $q>1$ then the series in question is periodic with frequency of $f=v / 2 \pi$, and a period $1 / f=2 \pi / v$.

\section{Data Description and Preliminary Statistics}

Daily adjusted closing price data was randomly chosen for the following seven indexes and one from commonly traded stocks from Newyork stock exchange. The data was collected from Yahoo finance website, apart from NS20 index data. We selected the most actively traded index-based data such as S\&P500, RUI(RUSSELL1000), CAC40, VIX, DAX, AMZN, and NSE20 index of Nairobi securities. Closing daily adjusted share prices were used to carry out the analysis. Table 1 provide summary of descriptive statistics of the data of daily log returns of the adjusted closing price.

Table 1. Summary Basic statistics of daily log returns

\begin{tabular}{rrrrrrrr}
\hline Basic.stats. & S\&P500 & VIX & CAC40 & RUI & DAX & NSE20 & AMZN \\
\hline No. $(n)$ & 6580.0 & 6584.0 & 6577.0 & 5835.0 & 6383.0 & 3651.0 & 4722.0 \\
& & & & & & & \\
Minimum & -0.0947 & -0.3506 & -0.0947 & -0.0956 & -0.0987 & -0.0523 & -0.2846 \\
Maximum & 0.1096 & 0.4960 & 0.1059 & 0.1104 & 0.1080 & 0.0695 & 0.2962 \\
1. Quartile & -0.0047 & -0.0360 & -0.0070 & -0.0048 & -0.0065 & -0.0036 & -0.0153 \\
3. Quartile & 0.0057 & 0.0318 & 0.0077 & 0.0057 & 0.0076 & 0.0036 & 0.0171 \\
& & & & & & & \\
Mean & 0.0002 & 0.0000 & 0.0001 & 0.0003 & 0.0003 & 0.0001 & 0.0012 \\
Median & 0.0005 & -0.0032 & 0.0003 & 0.0006 & 0.0008 & -0.0001 & 0.0000 \\
Sum & 1.6482 & 0.2273 & 0.7547 & 1.4866 & 1.8025 & 0.1871 & 5.7676 \\
SE Mean & 0.0001 & 0.0008 & 0.0002 & 0.0002 & 0.0002 & 0.0001 & 0.0006 \\
Variance & 0.0001 & 0.0040 & 0.0002 & 0.0001 & 0.0002 & 0.0001 & 0.0016 \\
Stdev & 0.0114 & 0.0629 & 0.0141 & 0.0117 & 0.0143 & 0.0085 & 0.0398 \\
Skewness & -0.2380 & 0.6692 & -0.0416 & -0.2717 & -0.1117 & 0.4454 & 0.4442 \\
Ex.Kurt & 8.5567 & 4.0910 & 4.3339 & 8.4719 & 4.5196 & 8.0967 & 7.5057 \\
& & & & & & & $16 \mathrm{May} 97$ \\
From & $03 J a n 90$ & $02 J a n 90$ & $03 J a n 90$ & $03 J a n 92$ & 26 Nov90 & $03 J u 195$ & 23 Feb16 \\
\hline
\end{tabular}

The total number of observations for the period of analysis is over 40,000 daily log returns as shown in Table 1. Whereas the population skewness and kurtosis for normal distribution are respectively 0 and 3 , statistics computed on log returns $X_{t}$, sample skewness and excess kurtosis are way far from zero and three respectively.

Figure 1, and Figure 2 displays autocorrelation of squared returns on the right and seasonal component extracted from the filtered but stationary, standardized residuals. It can be reported that for DAX index returns, filtered residues, the resulting spectral density is a constant which implies that the residuals represent a pure white noise as implied by equation (10). Table 2 reports the number of seasonal components observed across the other filtered data sets. For example based on NSE20 AR(3)GARCH(1,1) conditioned on students $\mathrm{t}$ distribution (7), filtered residuals, there seems to have four seasonality peaks of approximately 14,17,28, and 58 days respectively. Optimal ARCH models fit for changing variance is presented in the same table. For the other data sets the observed cycles are as presented in Figures 1 , and 2 respectively. 

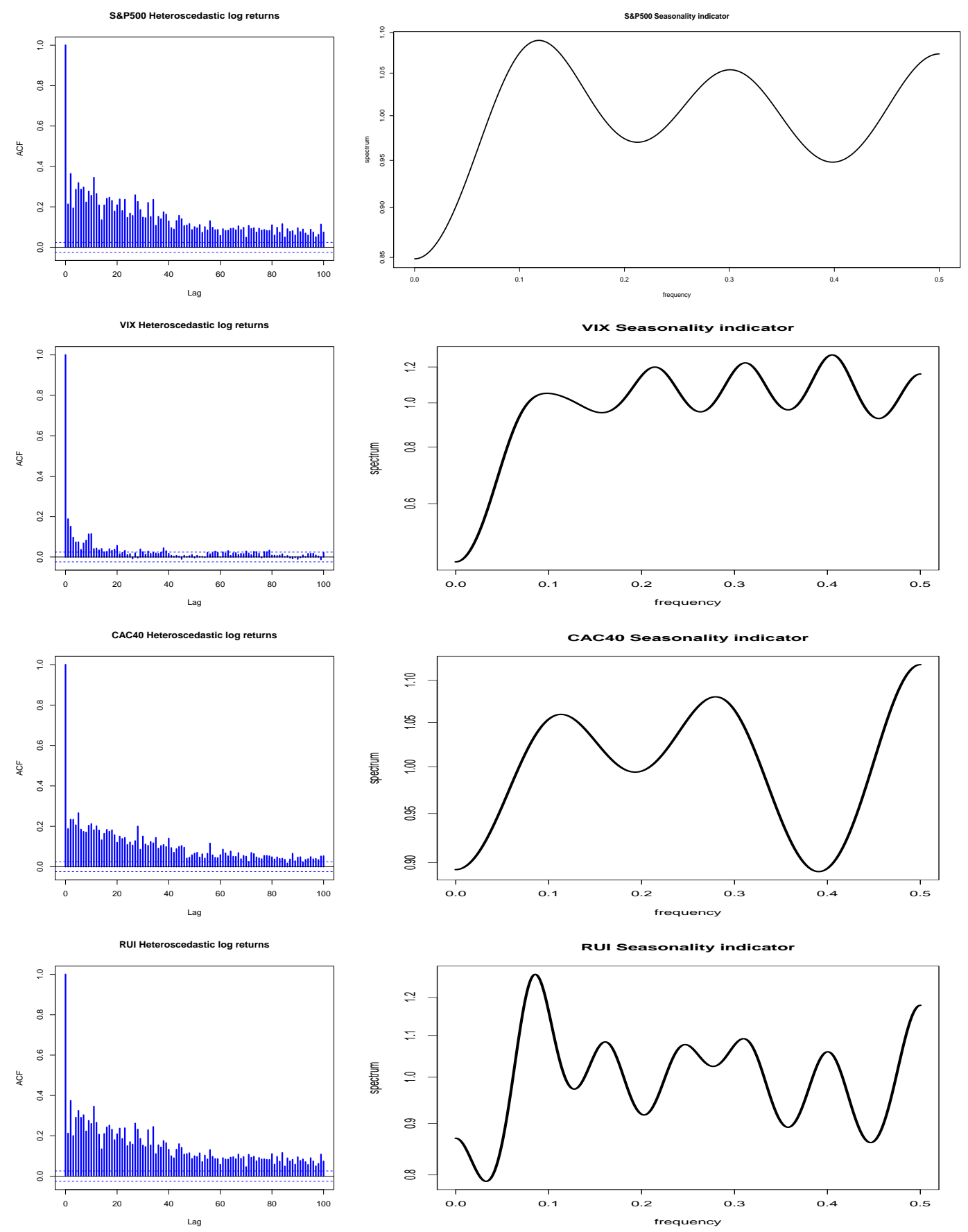

Figure 1. Analysis of returns seasonality for S\&P500, VIX, CAC40 and RUI 

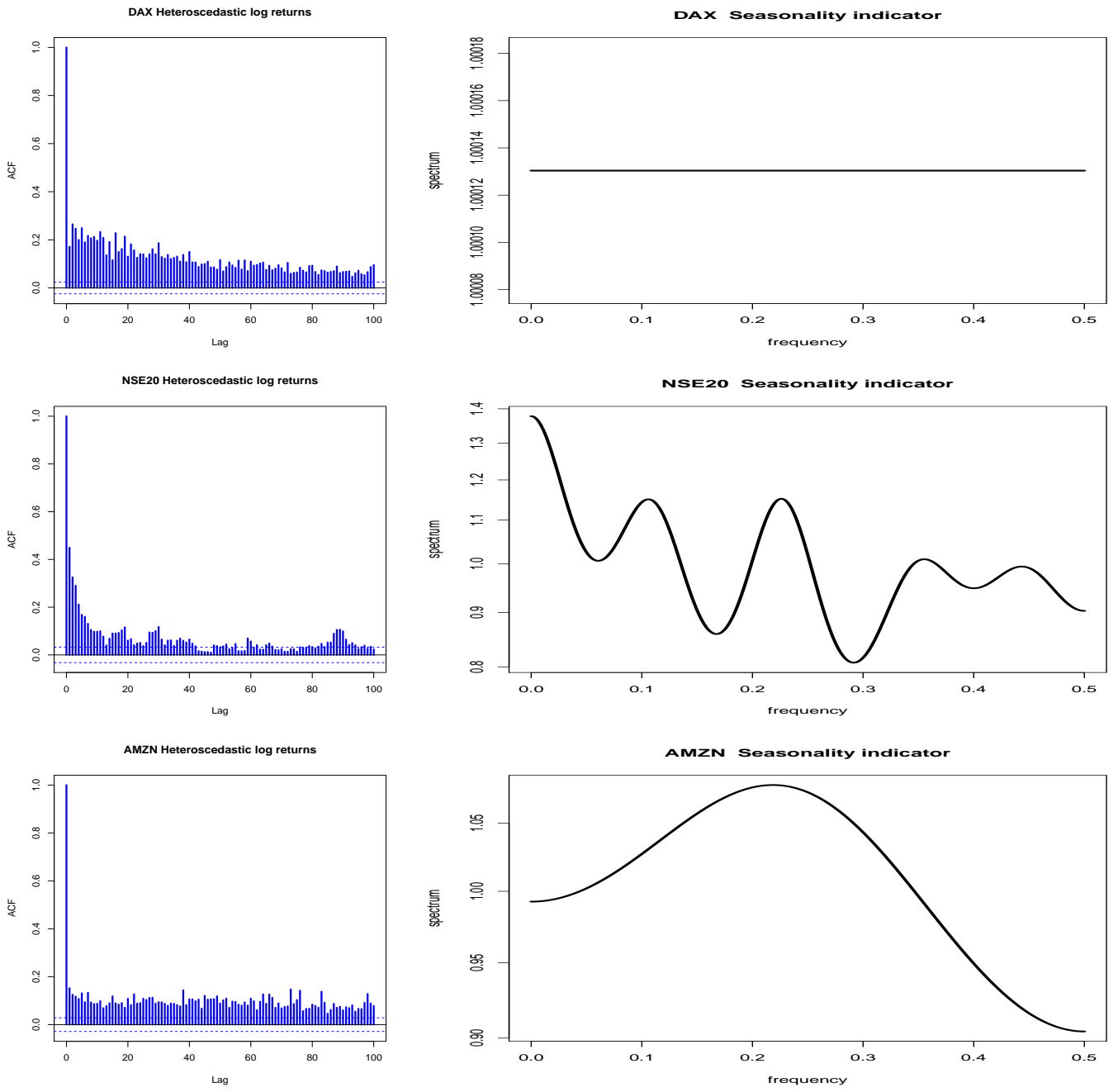

Figure 2. Analysis of returns seasonality for DAX,NSE20 and AMZN 
Table 2. ARMA-ARCH type models and spectral density results

\begin{tabular}{cccccc}
\hline \hline Param. $|\Phi|$ & max. \# & Market Index & $\mathrm{n}$ & Optimal model & Cond. distr. \\
\hline$|\Phi| \neq 0$ & 2 & S\&P500 & 6580 & $\operatorname{TARCH}(1,1)$ & $\operatorname{STD}(0,1)$ \\
$|\Phi| \neq 0$ & 4 & VIX & 6584 & $\operatorname{TGARCH}(1,1)$ & $\operatorname{NORM}(0,1)$ \\
$|\Phi| \neq 0$ & 2 & CAC40 & 6577 & $\operatorname{GARCH}(1,1)$ & $\operatorname{NORM}(0,1)$ \\
$|\Phi| \neq 0$ & 5 & RUI & 5835 & $\operatorname{TGARCH}(1,1)$ & $\operatorname{STD}(0,1)$ \\
$\Phi=0$ & 0 & DAX & 6383 & $\operatorname{GARCH}(1,1)$ & $\operatorname{NORM}(0,1)$ \\
$|\Phi| \neq 0$ & 4 & NSE20 & 3651 & $\operatorname{AR}(3)+\operatorname{GARCH}(1,1)$ & $\operatorname{STD}(0,1)$ \\
$|\Phi| \neq 0$ & 1 & AMZN & 3651 & $\operatorname{NGARCH}(1,1)$ & $\operatorname{NORM}(0,1)$ \\
\hline
\end{tabular}

Table 3 reports quasi-maximum likelihood estimates (QMLEs) using daily returns from respective indices. Optimal standard errors are reported in the parenthesis below the estimates. Loglik denotes the log likelihood value. All the standardized residual were non normal since their excess kurtosis were way far above zero. Q(5) is the Ljung-Box portmanteau test for up to 5th-order serial correlation in the standardized residuals, whereas $Q^{2}(5)$ is for up to 5 th order serial correlation in the squared standardized residuals. ARCH5 denotes the ARCH test.

\section{Concluding Remarks}

In this article we propose a feasible way of detecting seasonality within standardized residuals, after calibrating skewed time-varying volatility and leptokurtic log returns in discrete time within general framework of ARMA-ARCH Lévy model.Within the GARCH models framework, conditioned on either standardized student t distribution or standard normal were used to model changing variance. Assuming that the series had two contemporaneous components as proposed in [25] we further studied the standardized residuals using spectral density resolution to trace long term cycles present in the underlying process. If the changing variance is optimally eliminated then based on the basic principles of serial correlation and covariance stationarity, we examine all the data sets and where possible trace the underlying seasonality components. One can argue based of the evidence of the data set if there is any long term periodicity then the market data will review the signal, which was quite evident from the data set sampled. It will be more prudent if further empirical study will be done in the future to confirm or refute the observed results.

The proposed framework delivers predictive distribution which can be applied to option pricing of the payoff function for a given econometric model. As a result, the probability distribution could be useful to market participants who wish to compare the resulting model predictions to the potential prices on the market in developed and emerging economies. The framework takes care of most of the observed stylistic fact about financial time series data i.e. skewness and leptokurtic nature of demeaned GARCH filtered log returns, aggregational Gaussianity, and presence of jumps and in this case presence of seasonality.

a) Developed markets and emerging markets may not have the same underlying dynamics, but all the returns regardless of there origin are not normally distributed. It would be incorrect to assume that a universal model of log normal distribution would be ideal for all types of financial time series data sets

b) The presence of linear autoregressive dynamics, $\mathrm{AR}(3)-\mathrm{GARCH}(1,1)$ conditioned on student $\mathrm{t}$ distribution, reveals a seasonality signal of approximates $(7,15,28$ and 60$)$ working days as observed from the spectral density.

In this study we have not discussed whether presence of seasonality can be useful from a practitioners stand point. In addition, how one can exploit such information to implement a volatility trading strategy, was left for future research. see [29] for an interesting comparison of non random price movement.

Acknowledgements. The author would like to thank anonymous reviewers for their insightful comments. Financial assistance form ISP/EAUMP network is highly appreciated 
Table 3. Optimal model estimation result for each index and stock

\begin{tabular}{|c|c|c|c|c|c|c|c|}
\hline Index & S\&P500 & VIX & CAC40 & RUI & DAX & NSE20 & $\overline{A M Z N}$ \\
\hline Model & TGARCH & TGARCH & GARCH & TGARCH & GARCH & GARCH & NGARCH \\
\hline Loglik & 21683.1 & 9273.2 & 19555.1 & 19149.8 & 19040.9 & 13312.0 & 9419.9 \\
\hline$\mu$ & $\begin{array}{c}3.28 \mathrm{E}-04 \\
(8.7 \mathrm{E}-05)\end{array}$ & $\begin{array}{c}2.84 \mathrm{E}-03 \\
(7.1 \mathrm{E}-04)\end{array}$ & $\begin{array}{r}4.45 \mathrm{E}-04 \\
(1.36 \mathrm{E}-04)\end{array}$ & $\begin{array}{c}3.76 \mathrm{E}-04 \\
(1.1 \mathrm{E}-04)\end{array}$ & $\begin{array}{r}6.47 \mathrm{E}-04 \\
(1.35 \mathrm{E}-04)\end{array}$ & & $\begin{array}{r}1.48 \mathrm{E}-03 \\
(4.06 \mathrm{E}-04)\end{array}$ \\
\hline$\zeta_{1}$ & & & & & & $\begin{array}{r}0.2243 \\
(0.0180)\end{array}$ & \\
\hline$\zeta_{3}$ & & & & & & $\begin{array}{r}0.1332 \\
(0.0174) \\
0.0943 \\
(0.0165)\end{array}$ & \\
\hline$\omega$ & $\begin{array}{c}1.73 \mathrm{E}-04 \\
(3.6 \mathrm{E}-05)\end{array}$ & $\begin{array}{c}3.965 \mathrm{E}-03 \\
(4.87 \mathrm{E}-04)\end{array}$ & $\begin{array}{r}3.0 \mathrm{E}-06 \\
(1.0 \mathrm{E}-06)\end{array}$ & $\begin{array}{c}1.84 \mathrm{E}-04 \\
(3.3 \mathrm{E}-05)\end{array}$ & $\begin{array}{r}3.0 \mathrm{E}-06 \\
(1.0 \mathrm{E}-06)\end{array}$ & $\begin{array}{r}6.0 \mathrm{E}-06 \\
(1.0 \mathrm{E}-06)\end{array}$ & $\begin{array}{c}1.74 \mathrm{E}-04 \\
(2.0 \mathrm{E}-05)\end{array}$ \\
\hline$\alpha$ & $\begin{array}{r}0.07162 \\
(0.0102)\end{array}$ & $\begin{array}{r}0.07282 \\
(7.51 \mathrm{E}-03)\end{array}$ & $\begin{array}{r}0.0861 \\
(8.82 \mathrm{E}-03)\end{array}$ & $\begin{array}{r}0.0744 \\
(9.27 \mathrm{E}-03)\end{array}$ & $\begin{array}{r}8.32 \mathrm{E}-02 \\
(6.72 \mathrm{E}-03)\end{array}$ & $\begin{array}{r}0.2488 \\
(0.0199)\end{array}$ & $\begin{array}{r}3.41 \mathrm{E}-02 \\
(8.93 \mathrm{E}-04)\end{array}$ \\
\hline$\beta$ & $\begin{array}{r}0.9275 \\
(0.0107)\end{array}$ & $\begin{array}{r}0.883 \\
(0.0122)\end{array}$ & $\begin{array}{r}0.8978 \\
(9.55 \mathrm{E}-03)\end{array}$ & $\begin{array}{r}0.9244 \\
(9.43 \mathrm{E}-03)\end{array}$ & $\begin{array}{r}0.9002 \\
(7.68 \mathrm{E}-3)\end{array}$ & $\begin{array}{c}0.66154 \\
(0.0166)\end{array}$ & $\begin{array}{r}0.9695 \\
(2.96 \mathrm{E}-04)\end{array}$ \\
\hline$\gamma$ & & & & & & & $\begin{array}{r}1.0571 \\
(3.34 \mathrm{E}-02)\end{array}$ \\
\hline$\eta$ & $\begin{array}{r}0.969255 \\
(0.0961)\end{array}$ & $\begin{array}{r}-1.00 \\
(0.124)\end{array}$ & & $\begin{array}{r}0.9999 \\
(0.0981)\end{array}$ & & & \\
\hline$\nu$ & $\begin{array}{r}7.8939 \\
(0.7435)\end{array}$ & & & $\begin{array}{r}8.237 \\
(0.8744)\end{array}$ & & $\begin{array}{r}4.4072 \\
(0.2489)\end{array}$ & \\
\hline$Q(5)$ & $\begin{array}{r}4.519 \\
(0.1961)\end{array}$ & $\begin{array}{r}58.61 \\
(2.2 \mathrm{E}-16)\end{array}$ & $\begin{array}{r}4.897 \\
(0.1615)\end{array}$ & $\begin{array}{r}5.346 \\
(0.1276)\end{array}$ & $\begin{array}{r}3.285 \\
(0.3575)\end{array}$ & $\begin{array}{r}11.832 \\
(0.0 \mathrm{E}-0)\end{array}$ & $\begin{array}{r}6.335 \\
(0.0749)\end{array}$ \\
\hline$Q^{2}(5)$ & $\begin{array}{r}11.572 \\
(0.9269)\end{array}$ & $\begin{array}{r}3.145 \\
(0.3812)\end{array}$ & $\begin{array}{r}6.541 \\
(0.0669)\end{array}$ & $\begin{array}{r}11.159 \\
4.62 \mathrm{E}-03\end{array}$ & $\begin{array}{r}2.614 \\
(0.8206)\end{array}$ & $\begin{array}{r}2.077 \\
(0.8955)\end{array}$ & $\begin{array}{r}6.822 \\
(0.0573)\end{array}$ \\
\hline ARCH5 & $\begin{array}{r}1.4739 \\
(0.9269)\end{array}$ & $\begin{array}{r}0.622 \\
(0.8471)\end{array}$ & $\begin{array}{r}1.849 \\
(0.5055)\end{array}$ & $\begin{array}{r}1.584 \\
(0.5705)\end{array}$ & $\begin{array}{r}0.8365 \\
(0.7820)\end{array}$ & $\begin{array}{r}11.524 \\
(0.5859)\end{array}$ & $\begin{array}{r}0.2102 \\
(0.9631)\end{array}$ \\
\hline $\mathrm{AIC}$ & 6.588 & 2.816 & 5.945 & 6.5617 & 5.9649 & 7.2884 & 3.987 \\
\hline
\end{tabular}

\section{References}

1. S. J. Press, "A compound events model for security prices," Journal of business, pp. 317-335, 1967.

2. P. K. Clark, "A subordinated stochastic process model with finite variance for speculative prices," Econometrica: journal of the Econometric Society, pp. 135-155, 1973.

3. R. C. Merton, "Option pricing when underlying stock returns are discontinuous," Journal of financial economics, vol. 3, no. 1-2, pp. 125-144, 1976.

4. S. Beckers, "A note on estimating the parameters of the diffusion-jump model of stock returns," Journal of Financial and Quantitative Analysis, vol. 16, no. 01, pp. 127-140, 1981.

5. C. A. Ball and W. N. Torous, "On jumps in common stock prices and their impact on call option pricing," The Journal of Finance, vol. 40, no. 1, pp. 155-173, 1985.

6. P. Jorion, "On jump processes in the foreign exchange and stock markets," Review of Financial Studies, vol. 1, no. 4, pp. 427-445, 1988.

7. R. F. Engle, "Autoregressive conditional heteroscedasticity with estimates of the variance of united kingdom inflation," Econometrica: Journal of the Econometric Society, pp. 987-1007, 1982.

8. T. Bollerslev, "Generalized autoregressive conditional heteroskedasticity," Journal of econometrics, vol. 31, no. 3, pp. 307-327, 1986.

9. R. Cont, "Empirical properties of asset returns: stylized facts and statistical issues," Quantitave finance, vol. 1, pp. 223-236, 2001. 
10. T. H. Rydberg, "Realistic statistical modelling of financial data," International Statistical Review/Revue Internationale de Statistique, pp. 233-258, 2000.

11. E. Eberlein and U. Keller, "Hyperbolic distributions in finance," Bernoulli, pp. 281-299, 1995.

12. O. E. Barndorff-Nielsen, "Normal inverse gaussian distributions and stochastic volatility modelling," Scandinavian Journal of statistics, vol. 24, no. 1, pp. 1-13, 1997.

13. D. A. Hsieh, "The statistical properties of daily foreign exchange rates: 1974-1983," Journal of international economics, vol. 24, no. 1-2, pp. 129-145, 1988.

14. P. Christoffersen and K. Jacobs, "Which garch model for option valuation?" Management science, vol. 50, no. 9, pp. 1204-1221, 2004.

15. B. Eraker, M. Johannes, and N. Polson, "The impact of jumps in volatility and returns," The Journal of Finance, vol. 58, no. 3, pp. 1269-1300, 2003.

16. D. S. Bates, "Jumps and stochastic volatility: Exchange rate processes implicit in deutsche mark options," Review of financial studies, vol. 9, no. 1, pp. 69-107, 1996.

17. J. Pan, "The jump-risk premia implicit in options: Evidence from an integrated time-series study," Journal of financial economics, vol. 63, no. 1, pp. 3-50, 2002.

18. D. Duffie, J. Pan, and K. Singleton, "Transform analysis and asset pricing for affine jump-diffusions," Econometrica, vol. 68, no. 6, pp. 1343-1376, 2000.

19. W. H. Chan and J. M. Maheu, "Conditional jump dynamics in stock market returns," Journal of Business $\&$ Economic Statistics, vol. 20, no. 3, pp. 377-389, 2002.

20. L. Stentoft, "American option pricing using garch models and the normal inverse gaussian distribution," Journal of Financial Econometrics, vol. 6, no. 4, pp. 540-582, 2008.

21. I. J. Mwaniki, "Modeling heteroscedastic skewed and leptokurtic returns in discrete time," Journal of Applied finance and banking, vol. 9, no. 5, pp. 1-14, 2019.

22. G. Bakshi, C. Cao, and Z. Chen, "Empirical performance of alternative option pricing models," The Journal of finance, vol. 52, no. 5, pp. 2003-2049, 1997.

23. B. Dumas, J. Fleming, and R. E. Whaley, "Implied volatility functions: Empirical tests," The Journal of Finance, vol. 53, no. 6, pp. 2059-2106, 1998.

24. S. R. Das and R. K. Sundaram, "Of smiles and smirks: A term structure perspective," Journal of financial and quantitative analysis, vol. 34, no. 02, pp. 211-239, 1999.

25. J. M. Maheu and T. H. McCurdy, "News arrival, jump dynamics, and volatility components for individual stock returns," The Journal of Finance, vol. 59, no. 2, pp. 755-793, 2004.

26. R. F. Engle and V. K. Ng, "Measuring and testing the impact of news on volatility," The journal of finance, vol. 48, no. 5, pp. 1749-1778, 1993.

27. L. R. Glosten, R. Jagannathan, and D. E. Runkle, "On the relation between the expected value and the volatility of the nominal excess return on stocks," The journal of finance, vol. 48, no. 5, pp. 1779-1801, 1993.

28. J.-M. Zakoian, "Threshold heteroskedastic models," Journal of Economic Dynamics and control, vol. 18, no. 5, pp. 931-955, 1994.

29. D. B. Madan and K. Wang, "Nonrandom price movements," Finance Research Letters, 2016.

\section{Appendix}

\section{Log returns and ARCH models}

Stock price is assumed to be modelled by

$$
d S_{t}=\mu S_{t} d t+\sigma S_{t} d W_{t}, \quad \Rightarrow S_{T}=S_{t} \exp \left(\left(\mu-\frac{\sigma^{2}}{2}\right)(T-t)+\sigma\left(W_{T}-W_{t}\right)\right)
$$

Thus

$$
S_{T}=S_{t} \exp \left(X_{T}\right), \text { where } X_{T} \sim N\left[\left(\mu-\frac{\sigma^{2}}{2}\right)(T-t), \sigma^{2}(T-t)\right] .
$$

Let $T=t+1$, this implies that $\log _{e}\left(S_{t+1} / S_{t}\right)=X_{t}, \quad t=1,2, \ldots, n . X_{t}$ is known as $\log$ return, which can be positive or negative depending on the price dynamics. Recent studies assume that returns can be presented as

$$
X_{t}=\mu_{t}+\sigma_{t} z_{t}+\xi_{t+1}
$$

where $\mu_{t}$ is the mean component and $\sigma_{t} z_{t}$ represents the heteroscedastic part and the last term represents the residuals. Mean component could be modeled by ARMA models, while for the changing variance 
component, ARCH type models are widely used. If the residuals are i.i.d.'s, like in this study, then other properties could be investigated such as spectral analysis.

In general, [9] provides a broad review of the properties of returns known as stylized facts. One of the facts entails presence of changing variance $\sigma_{t}$ \{commonly known as volatility $\}$ inherent within the returns which are skewed and heavily tailed in distribution. The family of ARCH models provides a popular candidate for modeling heteroscedasticity, as found in [8] in general. let

$$
X_{t}=\mu+\varepsilon_{t} ; \quad \varepsilon_{t}=\sigma_{t} z_{t}, \quad z_{t} \sim i . i . d(0,1)
$$

The process $\left(\varepsilon_{t}\right), t \in \mathbb{Z}^{+} \quad$ is $\operatorname{ARCH}(q)$, where $\mathbb{E}\left[\varepsilon_{t} \mid \mathcal{F}_{t-1}\right]=0, \quad$ and $\operatorname{var}\left(\varepsilon_{t} \mid \mathcal{F}_{t-1}\right)=\sigma_{t}^{2}$, thus

$$
\sigma_{t}^{2}=\omega+\sum_{i=1}^{q} \alpha_{i} \varepsilon_{t-i}^{2} \quad \text { with } \omega>0, \alpha_{i} \geq 0, i=1,2, \ldots, q
$$

The $A R C H(q)$ model can be generalized by extending it with autoregressive terms of volatility. Some of the models studied in this paper include

$$
\begin{aligned}
\operatorname{GARCH}(\mathrm{p}, \mathrm{q}) \quad \sigma_{t}^{2} & =\omega+\sum_{i=1}^{q} \alpha_{i} \varepsilon_{t-i}^{2}+\sum_{j=1}^{p} \beta_{j} \sigma_{t-j}^{2} \\
\operatorname{TGARCH}(\mathrm{p}, \mathrm{q}), \sigma_{t}^{2} & =\omega+\sum_{i=1}^{q}\left[\alpha_{i}\left|\varepsilon_{t-i}\right|+\gamma_{i}\left|\varepsilon_{t-i}^{+}\right|\right]+\sum_{j=1}^{p} \beta_{j} \sigma_{t-j}^{2} \\
\operatorname{NGARCH}(1,1), \sigma_{t}^{2} & =\omega+\alpha \sigma_{t-1}^{2}+\beta \sigma_{t-1}^{2}\left(\epsilon_{t-1}+\gamma\right)^{2} .
\end{aligned}
$$

Quasi-Maximum likelihood approach is used to estimate the parameters of the ARCH models as shown in the Table 3 . 